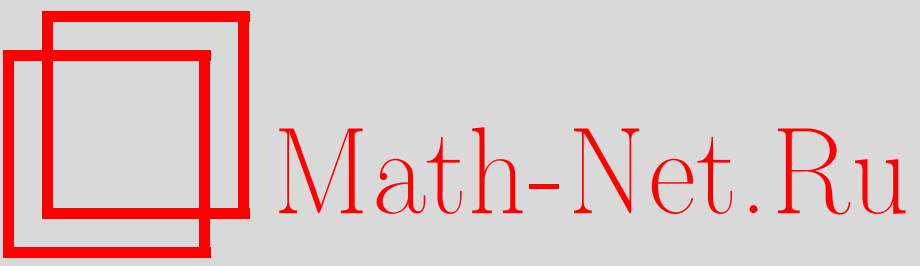

А. М. Вершик, Предельное распределение энергии квантового идеального газа с точки зрения теории разбиений натуральных чисел, УМH, 1997, том 52, выпуск 2, 139-146

DOI: https://doi.org/10.4213/rm823

Использование Общероссийского математического портала Math-Net.Ru подразумевает, что вы прочитали и согласны с пользовательским соглашением

http://www . mathnet.ru/rus/agreement

Параметры загрузки:

IP : 54.164 .48 .24

26 апреля 2023 г., 11:04:42 


\title{
ПРЕДЕЛЬНОЕ РАСПРЕДЕЛЕНИЕ ЭНЕРГИИ КВАНТОВОГО ИДЕАЛЬНОГО ГАЗА С ТОЧКИ ЗРЕНИЯ ТЕОРИИ РАЗБИЕНИЙ НАТУРАЛЬНЫХ ЧИСЕЛ
}

\author{
А. М. ВЕРшик
}

Р. Л. Добрушин был одним из тех, кто умел не только слушать “чужие” задачи, но и находить в них связь со своими проблемами. Я неоднократно обсуждал с ним темы, близкие к тому, о чем пойдет речь ниже. Беседы с ним всегда были интересны и полезны. Его математическое мьшление, по преимуществу вероятностное, позволяло ему четко увидеть собственно вероятностную часть проблемы, а таковая всегда присутствует в почти любой асимптотической задаче. Мы часто находили общее, обсуждая и далекие от математики вопросы. Его колоритная личность и математический талант привлекали друзей и молодежь, а сам он безусловно принадлежал к кругу тех, кто в значительной мере определял общую картину нашей математики.

0. Ставя и изучая задачи об асимптотиках и о предельных формах комбинаторных и геометрических объектов, автор часто оглядьвался на статистическую физику. Многие проблемы, из которых возникают подобные задачи (случайные разбиения, подстановки, асимптотическая теория представлений, рост конфигураций, статистическая геометрия, теория чисел и т. п.), имеют недвусмысленно статфизический характер. Эти задачи разнообразны по мотивировкам, ответам и связям с другими областями математики. В то же время, помимо собственного интереса они, видимо, должны играть роль полезных алгебраических и геометрических моделей для более сложных проблем (таких, как фазовые переходы в многомерных системах и др).

Цель этой работы в том, чтобы показать, насколько близки две задачи - о типичной асимптотической форме разбиения натурального числа и о вьчислении предельного распределения энергии квантового идеального газа. Мы приводим решение первой и истолковываем его в терминах второй. Нет сомнения в полезности этого параллелизма для обеих областей.

1. Задача о распределении энергии в большом и малом каноническом ансамблях квантового идеального газа с той или иной статистикой замечательньм образом включается в широкий класс проблем, к которому относятся задачи аддитивной асимптотической теории чисел с неограниченно растушим числом слагаемых, в частности, задачи о разбиениях натуральных чисел и векторов. В традищионной теории интересуются, как правило, числом или асимптотикой числа решений (разбиений). Нас же интересует более обший вопрос - об асимптотической структуре типичных разбиений 
или конфигураций, об их предельной форме, инвариантах и др. В аддитивной теории чисел, как правило, число слагаемых фиксировано (например, в классической проблеме Варинга), но в задачах комбинаторики, геометрии, не говоря уже о статистической физике, число слагаемых растет заданным или случайным образом.

Вот одна из формулировок такого рода. Рассматривается счетное множество $\Lambda \subset \mathbb{R}_{+}$без конечных предельных точек и число $E \in \mathbb{R}_{+}$; на множестве всех разложений числа $E: E=\lambda_{1}+\lambda_{2}+\cdots, \lambda_{i} \in \Lambda$, упорядоченных по убьванию: $\lambda_{1} \geqslant \lambda_{2} \geqslant \ldots$, задается мера (статистика) и ставится вопрос об асимптотических свойствах таких мер, когда $E \rightarrow \infty$. Сушествует ли (после необходимой нормировки разложений) их предел в том или ином смысле; если “да”, то вырожден он или нет? То есть являются ли почти все разложения асимптотически одинаковыми?

Можно заменить $\mathbb{R}_{+}$на $\mathbb{R}_{+}^{n}$ или на коммутативную полугрупп, а $\Lambda$ - на то или иное подмножество в ней; можно налагать различные дополнительные условия на слагаемые и т.п. (рост их числа, размеры и др.). Задача об асимптотике мер и, в частности, о предельной форме ("limit shape") является ключевой проблемой, она связана со многими областями математики см. [1], [2].

2. Рассмотрим более подробно задачи о разбиениях натуральных чисел. В этом случае $\Lambda=\mathbb{N}, E \equiv n \in \mathbb{N}$ в предыдуших обозначениях. Будем обозначать множество всех разбиений натурального числа $n$ через $\mathscr{P}_{n}=\{\lambda: \lambda \vdash n\}$, а сумму для данного разбиения $\lambda$ как $n(\lambda)=n$, обозначим также $\mathscr{P}=\bigcup_{n} \mathscr{P} n$. Для данного разбиения $\lambda=\left\{\lambda_{i}\right\}$ обозначим $r_{k}(\lambda)=\#\left\{i: \lambda_{i}=k\right\}$ (число слагаемых, равных данному числу) и назовем их числами заполнения. Очевидно, $\sum_{k} k r_{k}(\lambda)=n(\lambda)$, а число слагаемых есть $\sum_{k} r_{k}(\lambda) \equiv N(\lambda)$. Обозначим через $\mathscr{P}_{n, N}=\{\lambda: n(\lambda)=n, N(\lambda)=N\}$ множество разбиений числа $n$ с $N$ слагаемьми. Распределением разбиения $\lambda$ назовем функцию $\varphi_{\lambda}: \mathbb{R}_{+} \rightarrow \mathbb{N}$

$$
\varphi_{\lambda}(t)=\sum_{k=[t]}^{\infty} r_{k}(\lambda)
$$

ее подграфик есть диаграмма Юнга. Очевидно, $\varphi_{\lambda}(0)=N(\lambda), \int_{0}^{\infty} \varphi_{\lambda}(t) d t=n(\lambda)$.

Выделим важный класс мер на разбиениях: семейство статистик (мер) $\mu_{x}$ на $\mathscr{P}$ назовем мультипликативнылм, если

1) числа заполнения $\left\{r_{k}(\cdot)\right\}_{k=0}^{\infty}$ как функции на пространствах с мерой $\left(\mathscr{P}, \mu_{x}\right)$ независимы для всех мер $\mu_{x}$;

2) ограничения мер $\mu_{x}$ на $\mathscr{P}_{n}, n=1,2, \ldots$, не зависят от $x$ :

$$
\left.\frac{1}{\mu_{x}(\mathscr{P})} \mu_{x}\right|_{\mathscr{P}_{n}} \equiv \mu^{n}
$$

ЛеммА. Заменой параметра всякое мультипликативное семейство приводится $к$ следующему виду: параметр х пробегает открытый интервал $\left(0, R_{0}\right)$, $R_{0} \leqslant \infty$; существуют функции $\mathscr{F}_{k}(\cdot)$, аналитические в круге $|z|<R_{0}$ и имеющие неотрицательные коэффичиенты тәйлора $\mathscr{F}_{k}(y)=\sum_{r=0}^{\infty} c_{k r} y^{r}, k=1,2, \ldots$, $c_{k r} \geqslant 0$, при әтом

$$
\begin{gathered}
\mu_{x}(\{\lambda\})=x^{n(\lambda)} \prod_{k} \mathscr{F}_{k}\left(x^{k}\right)^{-1} c_{k r_{k}(\lambda)}, \\
\mu_{x}\left(\{\lambda\}: r_{k}(\lambda)=r\right)=c_{k r} x^{k r} \mathscr{F}_{k}\left(x^{k}\right)^{-1} .
\end{gathered}
$$




\section{ЗАМЕЧАНИЯ.}

1. Из сравнения вьшеприведенных формул вытекает независимость функций $r_{1}(\lambda), r_{2}(\lambda), \ldots$.

2. Лемма утверждает, что мультипликативное семейство задается одной аналитической в круге $|z|<R_{0}$ функцией $\mathscr{F}(x)$ и ее разложением в бесконечное произведение $\mathscr{F}(x)=\prod_{k=1}^{\infty} \mathscr{F}_{k}\left(x^{k}\right)$, где все сомножители $\mathscr{F}_{k}$ имеют неотрицательные коэффициенты Тэйлора. Функция $\mathscr{F}_{k}$ есть производящая функция распределения величины $r_{k}(\cdot)$.

3. Нормированные ограничения $\mu_{x}$ на $\mathscr{P}_{n}$ вьглядят так:

$$
\mu^{n}(\{\lambda\}) \equiv \frac{1}{\mu_{x}\left(\mathscr{P}_{n}\right)} \mu_{x}(\{\lambda\})=Q_{n}^{-1} \prod_{k} c_{k r_{k}(\lambda)},
$$

где $Q_{n}=\sum_{\lambda \vdash n} \prod_{k} c_{k r_{k}(\lambda)}$, при этом мера $\mu_{x}$ есть вьпуклая комбинация мер $\mu^{n}$ :

$$
\mu_{x}=\sum_{n=0}^{\infty} x^{n} Q_{n} \mathscr{F}(x)^{-1} \mu^{n} .
$$

Те же данные $\left(\mathscr{F}(x)=\prod_{k} \mathscr{F}_{k}\left(x^{k}\right)\right)$ задают мультипликативное семейство на малом каноническом ансамбле - $\mathscr{P}_{n, N}$. В этом случае формулы таковы:

$$
\begin{gathered}
\mu_{x, z}(\{\lambda\})=x^{n(\lambda)} \cdot z^{N(\lambda)} \cdot \prod_{k} \mathscr{F}_{k}\left(z x^{k}\right)^{-1} c_{k r_{k}(\lambda)} ; \\
\mu^{n, N}(\{\lambda\}) \equiv \frac{1}{\mu_{x, z}\left(\mathscr{P}_{n, N}\right)} \mu_{x, z}(\{\lambda\})=Q_{n, N}^{-1} \prod_{k} c_{k r_{k}(\lambda)},
\end{gathered}
$$

где

$$
Q_{n, N}=\sum_{\substack{\lambda \perp n \\ N(\lambda)=N}} \prod_{k} c_{k r_{k}(\lambda)} \quad \text { (малая статсумма) }
$$

Аналогии с понятиями статфизики идеального газа здесь очевидны - см. о них далее.

Основная проблема теперь ставится так: найти асимптотическое поведение мер $\mu^{n}, \mu^{n, N}$ при $n, N \rightarrow \infty$ и мер $\mu_{x}, \mu_{x, z}$ при $x \rightarrow R_{0}, z \rightarrow 1$ и сравнить $u x$. Совпадение пределов этих мер означает асимптотическую эквивалентность ансамблей $\left(\mathscr{P}, \mu_{x}\right)$ и $\left(\mathscr{P}_{n}, \mu^{n}\right)$ или $\left(\mathscr{P}, \mu_{x, z}\right)$ и $\left(\mathscr{P}_{n, N}, \mu^{n, N}\right)$. Вопрос об асимптотике включает в себя нахождение подходящей нетривиальной нормировки (скейлинга), позволяющей рассматривать все меры в одном масштабе. Этот, в существенном единственный, скейлинг и прямой ответ на поставленньй выше вопрос в случае классических статистик для квантового идеального газа и их обобщений будет дан ниже.

3. Из всего обширного класса мультипликативных мер рассмотрим лишш следующий:

$$
\mathscr{F}_{\left\{b_{k}\right\}}^{B E}(x)=\prod_{k=1}^{\infty} \frac{1}{\left(1-x^{k}\right)^{b_{k}}}, \quad \mathscr{F}_{\left\{b_{k}\right\}}^{F D}(x)=\prod_{k=1}^{\infty}\left(1+x^{k}\right)^{b_{k}},
$$

где $b_{k} \in \mathbb{N}, k=1,2, \ldots$ В силу предыдущего эти функции задают статистики на $\mathscr{P}$, $\mathscr{P}_{n}, \mathscr{P}_{n, N}-\mu_{x,\left\{b_{k}\right\}}$ и т. д. Мы опускаем указание на $\left\{b_{k}\right\}$, когда это возможно. Если 
$b_{k}=j_{d}(k)=\#\left\{\left(k_{1}, \ldots, k_{d}\right) \in \mathbb{Z}^{d}: k_{1}^{2}+\cdots+k_{d}^{2}=k\right\}$, то эти статистики совпадают со статистиками Бозе-Эйнштейна и Ферми-Дирака $d$-мерного квантового идеального газа.

Определим число $\alpha=\alpha\left(\left\{b_{k}\right\}\right)$ :

$$
\begin{gathered}
\alpha=\inf \left\{\alpha^{\prime}: \text { ряд Дирихле } \sum_{k=1}^{\infty} \frac{b_{k}}{k^{s}}\right. \text { есть аналитическая } \\
\text { функция от } \left.s \text { в полуплоскости } \operatorname{Re} s \geqslant \alpha^{\prime}\right\} .
\end{gathered}
$$

Для $b_{k}=j_{k}(d)$ по известной теореме Зигеля $\alpha=d / 2$.

Параметр $\alpha$ - единственная характеристика функций $\mathscr{F} B E$ и $\mathscr{F} F D$, которая нужна для ответов на поставленные вопросы. Мы будем считать, что $\alpha<\infty$.

Нижеследуюшая теорема утверждает, что существует и притом единственный скейлинг, при котором меры $\mu_{x}$ и $\mu^{n}$ сходятся, и предел есть вырожденная мера, сосредоточенная на одной замечательной кривой, однозначно определяемой по числу $\alpha$.

Tеорема. Для любых $a, b>0 u \varepsilon>0$

$$
\begin{aligned}
& \lim _{x \rightarrow 1} \mu_{x}\left\{\lambda \in \mathscr{P}: \sup _{t \in[a, b]}\left|n^{-\frac{\alpha}{\alpha+1}} \sum_{k \geqslant t n \frac{1}{1+\alpha}} r_{k}(\lambda)-\int_{t}^{\infty} u^{\alpha-1} \frac{e^{-c u}}{1 \mp e^{-c u}} d u\right|<\varepsilon\right\}=1, \\
& \lim _{n \rightarrow \infty} \mu^{n}\left\{\lambda \vdash n: \sup _{t \in[a, b]}\left|n^{-\frac{\alpha}{\alpha+1}} \sum_{k \geqslant t n^{\frac{1}{+\alpha}}} r_{k}(\lambda)-\int_{t}^{\infty} u^{\alpha-1} \frac{e^{-c u}}{1 \mp e^{-c u}} d u\right|<\varepsilon\right\}=1 .
\end{aligned}
$$

Выбор знака Ғ отвечает статистикам $B E$ и $F D$, соответственно, константа с определена ниже.

Таким образом, скейлинг с точностью до констант определяется так: $\left(n^{\frac{1}{\alpha+1}}, n^{\frac{\alpha}{\alpha+1}}\right)$; предельные меры на обоих ансамблях совпадают и являются $\delta$-мерой на кривой, определяемой уравнением

$$
\Gamma_{\alpha}(t)=\int_{t}^{\infty} u^{\alpha-1} \frac{e^{-c u}}{1 \mp e^{-c u}} d u
$$

Функция $\Gamma_{\alpha}(t)$ есть плотность распределения, поэтому константа $c$ определяется из условия

$$
\int_{0}^{\infty} \Gamma_{\alpha}(t) d t=1
$$

Заметим, что для $\alpha>1$ (т.е. $d>2$ ) $\Gamma_{\alpha}(0)<\infty$, а для $0<\alpha \leqslant 1 \Gamma_{\alpha}(0)=\infty$. Случай $\alpha=1$ соответствует, с одной стороны, равномерному распределению на $\mathscr{P}_{n}$ и эйлеровым функциям

$$
\mathscr{F}^{B E}(x)=\prod_{k=1}^{\infty} \frac{1}{1-x^{k}}, \quad \mathscr{F}^{F D}(x)=\prod_{k=1}^{\infty}\left(1+x^{k}\right),
$$


а с другой - двумерному идеальному газу $(\alpha=d / 2)$. Кривая $\Gamma_{1}(t)$ в симметричной форме записьвается уравнением (ВЕ-случай)

$$
\exp \left(-\frac{\pi}{\sqrt{6}} x\right)+\exp \left(-\frac{\pi}{\sqrt{6}} y\right)=1
$$

а скейлинг $-(\sqrt{n}, \sqrt{n})$.

Любопытно, что ее поведение в нуле (логарифмическая особенность) объясняет старую теорему П. Эрдеша о том, что в типичном разбиении числа $n$ число слагаемых растет как $c \sqrt{n} \ln n$, что имеет прямое отношение к вопросу о фазовом переходе (точнее, его отсутствию при $d \leqslant 2-$ см. далее).

Фиксашия роста числа слагаемых приводит к аналогичной теореме для мер $\mu^{n, N}$, а именно, если $N=v n^{\frac{\alpha}{1+\alpha}}, v>0$, то соответствуюшая формула для $\Gamma_{\alpha, v}$ при $\alpha \leqslant 1$ такова:

$$
\Gamma_{\alpha, v}(t)=\int_{t}^{\infty} u^{\alpha-1} \frac{e^{-c u}}{1 \mp v e^{-c u}} d u
$$

Если $\alpha>1$, то при достаточно больших $v$ к графику кривой добавляется атом в 0 ("конденсация Бозе-Эйнштейна" - см. далее). Кривые $\Gamma_{\alpha}, \Gamma_{\alpha, v}$ есть пределы диаграмм Юнга в подходяшей нормировке, т.е. предельные распределения разбиений. Функции $\Gamma_{\alpha}$ и $\Gamma_{\alpha, v}$ содержат информацию о главном члене асимптотик любых функционалов от типичных разбиений.

4. Остановимся кратко на основном вопросе - связи со статистикой идеального квантового газа. Автору была полезна конструктивная критика Л.А. Халфина, способствовавшая уточнению некоторых соответствий.

Состояние (= точка ансамбля = конфигурация) определяется в этом случае набором импульсов частиц, входяших в конфигурацию, т.е. набором собственных значений (с кратностями) задачи Дирихле для оператора Лапласа с периодическими граничными условиями. Пусть $V$ - объем $d$-мерного тора, $\omega-$ состояние системы. Тогда энергия состояния задается формулой

$$
E_{\omega}=\frac{(2 \pi \hbar)^{2}}{2 m} \sum_{q \in \omega \subset \mathbb{Z}^{d}} \frac{1}{V^{\frac{2}{d}}}\|q\|^{2},
$$

где $\|\cdot\|-d$-мерная евклидова норма. Приняв систему единиц, в которой множитель перед суммой равен 1 , мы получаем с точностью до обозначений ситуацию, рассмотренную выше, в которой

$$
\mathscr{F}(x)=\prod_{k=1}^{\infty} \frac{1}{\left(1-x^{k}\right)^{j_{d}(k)}}
$$

(ВЕ-статистика).

Асимптотики всех величин (число частии, обвем) соотносятся с ростом энергии, которая является для нас основным параметром; в этом, быть может, некоторое методическое отличие от традиции. Грубо говоря, энергия и есть то натуральное число, которое мы разбиваем: $E=n$. Слагаемые, с точностью до множителя (в который входит обратная температура), есть натуральные числа, поделенные на некоторую степень объема. 
Из приведенной выше теоремы следует, что скейлинг по осям с точносью до констант таков:

$$
\left(n^{\frac{1}{\alpha+1}}, n^{\frac{\alpha}{\alpha+1}}\right)=\left(E^{\frac{2}{d+2}}, E^{\frac{d}{d+2}}\right)
$$

напомним, что $\alpha=d / 2$. Поэтому $V=V(E) \simeq E^{\frac{d}{d+2}}, N=N(E) \simeq E^{\frac{d}{d+2}}$ (по порядку). Здесь мы рассматриваем случай, в котором число частищ не фиксируется, а является случайным (химический потенциал равен нулю). Константа в скейлинге не имеет значения, когда речь идет о мерах $\mu^{n}$, но для скейлинга мер $\mu_{x}$ константа уже существенна (см. ниже) - она зависит от $x$, а $x$ в статфизической интерпретации есть экспонента от обратной температуры со знаком минус, деленной на нужную степень объема.

Простой подсчет показывет, что соотношение между объемом (т.е. между размером слагаемых или, иначе, энергией частиц), с одной стороны, и числом частиц, с другой, автоматически устанавливается таким, каким оно должно быть при термодинамическом предельном переходе. Таким образом, наш предельный переход в точности соответствует термодинамическому пределу, в котором $N$ и $V$ имеют одинаковый порядок роста. Если же зафиксировать предел отношения числа частиц (слагаемых) к объему, то мы приходим к аналогичной проблеме для условного распределения, т.е. для малого канонического ансамбля - см. далее.

Заметим, что в теоретико-числовых и геометрических задачах уместно рассматривать ситуации, когда соотношение между $V=V(E)$ и $N=N(E)$, т.е. между числом и размером слагаемых или суммой, необязательно столь жесткое, как в термодинамическом пределе. Например, число слагаемых может быть заранее заданной функцией суммы.

Вернемся к статфизической интерпретации теоремы, сформулированной выше. Пусть $r_{k}(\omega)$ - число частищ с энергией, равной $k$, т.е. сумма обычных чисел заполнения по сфере радиуса $k$ в импульсной решетке. Сформулируем теорему в подходящих терминах. Пусть $\varepsilon>0$.

ТеОрема. При достаточно больиих энергиях $E>E_{\varepsilon}$ множество состояний идеального ВЕ-газа (число частии, случайно), для которых выполнено нижеследующее свойство (*), имеет гиббсовскую меру, бо́льшую $1-\varepsilon$ :

$$
\sup _{0<a \leqslant t \leqslant b<\infty}\left|E(\omega)^{-\frac{d}{d+2}} . \sum_{k \geqslant t E^{\frac{2}{d+2}}} r_{k}(\omega)-\Gamma_{\frac{d}{2}}(t)\right|<\varepsilon .
$$

Таким образом, кривая $\Gamma_{\frac{d}{2}}$ есть графики предельной плотности распределения $E(\omega)$ - энергии типичной конфигурации по нормированной на $E^{\frac{2}{d+2}}$ энергии частиц.

Отсюда следует, например, что в типичной конфигурации суммарная энергия всех частиц, каждая из которых имеет энергию, лежащую в интервале $\left(a E^{\frac{2}{d+2}}, b E^{\frac{2}{d+2}}\right)$, асимптотически равна $\int_{a}^{b} \Gamma_{\alpha}(t) d t \cdot E$, а число частиц с энергией, бо́льшей, чем $t E^{\frac{2}{d+2}}$, равно $\Gamma_{\alpha}(t) E^{\frac{2}{d+2}}$.

Принципиально важным является вопрос о конечности $\Gamma_{\alpha}(0)$ : если это число конечно, то число частищ (слагаемых) в типичной конфигурации растет, как $\Gamma_{\alpha}(0) E^{\frac{2}{d+2}}$, 
если же бесконечно, то чуть (на степень логарифма) быстрее. Заметим, что все формулы имеют смысл и для дробных размерностей $d$; в этом случае $b_{k}=\left[k^{\alpha-1}\right]$, если $1 \leqslant \alpha$ и, по-прежнему, $d=2 \alpha$; значение нашей плотности в нуле конечно в размерностях $d>2$.

Приведем более явную формулу для $d=3$ - трехмерного идеального ВЕ-газа:

$$
\Gamma_{\frac{3}{2}}(t)=\int_{t}^{\infty} \sqrt{u} \frac{e^{-c u}}{1-e^{-c u}} d u, \quad c=\left[\Gamma\left(\frac{5}{2}\right) \zeta\left(\frac{5}{2}\right)\right]^{\frac{2}{5}} .
$$

Здесь Г есть гамма-функция, а $\zeta$ - дзета-функция Римана. В нуле плотность распределения $\Gamma_{\alpha}(t)$ ограниченна.

О размерности $d=2(\alpha=1)$ мы уже говорили - этот случай в точности совпадает с классическим равномерным распределением на разбиениях. Функция $\Gamma_{1}(\cdot)$ была вьписана вьше. Ее значение в нуле неограниченно. Асимптотики многих конкретных функционалов найдены П.Эрдешем и его последователями, но связи со статфизикой, как будто бы, не отмечались, как и не ставилась задача о предельной форме. Теорема Эрдеша о типичном числе слагаемых в равномерно распределенных разбиениях натурального числа $(c \sqrt{n} \ln n)$ становится теоремой о типичном числе частиц, т.е. об асимптотике $\Gamma_{\alpha}$ в нуле. Она также может быть выведена из теоремы о предельной форме.

С теоретико-числовой точки зрения любопытен и одномерный случай $d=1$, не представляюший, конечно, интереса для статфизики; здесь $\alpha=\frac{1}{2}$, а $b_{k}=1$, если $k$ есть полный квадрат, и $b_{k}=0$, если $k$ не есть полньй квадрат, - он отвечает задаче о предельной плотности разбиения натурального числа в сумму квадратов с неограниченньм числом слагаемых - иначе говоря, это неограниченная квадратичная проблема Варинга; ответ здесь также дается формулой, приведенной в теореме при значении $\alpha=\frac{1}{2}$.

Для комбинаторики очень интересна размерность $d=4, \alpha=2$, это - асимптотическая теория плоских разбиений или трехмерных диаграмм Юнга; о ней пойдет речь в другой работе.

Если мы зафиксируем рост числа слагаемых (частиц) в естественном диапазоне $c$ точностью до константы $-N \sim c E^{\frac{d}{d+2}}$ (а тогда, как уже упоминалось, автоматически фиксируется и $\frac{N}{V} \rightarrow v$, где $v$ - конечная плотность), то ответ также может быть получен аналогичными методами. Здесь речь идет уже о малом каноническом ансамбле и о слабых пределах при надлежашем скейлинге мер $\mu_{x, z}$ и $\mu^{n, N}$; в этом случае химический потенциал отличен от нуля. Пределы этих мер могут и не совпадать, даже если соотношения между взаимным ростом $N, n$ и скоростью взаимного стремления к 1 переменных $x, z$ согласована - см. [3]. Слабый предел мер $\mu^{n, N}$ снова даст $\delta$-меру, сосредоточенную или на некоторой кривой, или на кривой с дополнительньм атомом в нуле (см. вьше).

Различие между размерностями (в том числе и дробными) $d>2$ и $d \leqslant 2$ (т.е. между $\alpha>1$ и $\alpha \leqslant 1$ ) выражается в наличии әффекта конденсации Бозе-Эйнштейна, суть которого в наших терминах объясняется так. Если $\Gamma_{\alpha}(0)=\infty$ (а это так в размерностях $d \leqslant 2$ ), то любое заданное конечное значение числа частиц, т. е. слагаемых, (нормированного на нужную степень энергии) будет меньше по порядку, чем число частиц, в том случае, когда оно случайно. Действительно, последнее для типичной 
конфигурации, как отмечалось вьше, растет с ростом энергии $E$ несколько быстрее, чем $E^{\frac{d}{d+2}} \cdot$, что, собственно, и означает бесконечность $\Gamma_{\alpha}(0)$. В этом случае предел мер $\mu^{n, N}$ в нужном скейлинге конщентрируется на кривой - плотность распределения энергии, формула которой приводилась. Если же $\Gamma_{\alpha}(0)<\infty$ (т.е. $d>2$ ), то при заданном нормированном числе частищ (слагаемых) бо́льшем, чем $\Gamma_{\alpha}(0)$, "заказанное" число будет больше, даже чем в случае, когда число частиц случайно, "недостающее” число частиц может быть восполнено только за счет частиц с нулевьм импульсом, т.е. за счет нулевых слагаемых, и предел мер $\mu^{n, N}$ будет сосредоточен на кривой с атомом в нуле - в этом и состоит явление конденсации Бозе-Эйнштейна: доля нулевых частиц с нулевым импульсом (нулевых слагаемых) - положительна.

Это объяснение слегка отличается от обычных (см. [4]) той ролью, которую играет случай нулевого химпотенциала (случайного числа частиц): мы сравниваем значения $\Gamma_{\alpha}(0)$ и нормированного числа частиц при фиксированной плотности. Напомним, что в нормировку числа частиц и, тем самым, функции $\Gamma_{\alpha}(\cdot)$ и ее значения в нуле неявно входит температура (см. вьше - скейлинг был определен с точностью до константы). Поэтому вьшеприведенное рассуждение в конще конщов приводит в обычному критерию: параметр конденсации определяется тем же известным выражением от плотности и температуры (см., например, [4; формула (55.5)]), которое и следует сравнивать с нашим $\Gamma_{\alpha}(0)$.

С другой стороны, ничего, кроме комбинаторных терминов, связанных с разбиениями натуральных чисел, во всем предыдущем, в частности в объяснении конденсации Бозе-Эйнштейна, не использовалось. Таким образом, конденсацию Бозе-Эйнштейна в равной мере можно “наблюдать" и в статфизике, и в этой, а также подобных ей, комбинаторно-числовых задачах. Я думаю, что имеется много таких обоюдополезных пересечений.

Кривые $\Gamma_{\alpha}$ являются экстремалями некоторых вариационных задач. Соответствующий вариационньй принцип сфформулирован автором (см. [1], [2]), но подробнее будет обсужден в другом месте. Вариационньй принцип тесно связан с методом больших уклонений и энтропией. Р. Л. Добрушин был одним из пионеров применения этих методов в статистической физике.

\section{СПИСОК ЛИТЕРАТУРЫ}

[1] Вершик А. М. Статистическая механика комбинаторных разбиений и их предельные формы // Функц. анал. и его прил. 1996. Т. 30. № 2. С. 19-39.

[2] Vershik A.M. Asymptotic combinatorics and algebraic analysis // Proceedings of the International Congress of Mathematicians, Zürich, 1994. V. 2. Basel: Birkhäuser, 1995. P. 1384-1394.

[3] Lewis J. T. The Free Boson Gas // LMS Sympos. Math. Contemp. Phys. New York: Acad. Press, 1972. P. 209-226.

[4] Ландау Л.Д., Лифшиц Е. М. Статистическая физика. М.: Наука, 1964.

С.-Петербургское отделение

Поступила в редакцию

Математического института РАН

15.09.1996 